\title{
In-situ and laboratory high-pressure cultivations of deep microbiome at two Japanese underground facilities
}

\author{
Y. SUZUKI ${ }^{1 *}$
}

${ }^{1}$ Earth \& Planetary Sci. Dept., The Univ. of Tokyo, Tokyo 113-0033, Japan (*correspondence: yoheysuzuki@eps.s.u-tokyo.ac.jp)

Underground Fasilitiy: New Frontier for Deep Mirobiome It remains enigmatic whether the doubling time of subsurfae microbiome tends to range from thousands to millions of years based on biogeochemical profiles [1]. In contrast, laboratory-based activity measurements are considered to overestimate metabolic rates, given technical difficulties in reproducing subsurface conditions such as high pressure and extremely low energy fluxes.

In this study, in-situ cultivation was performed for granite-hosted microbiome in a 300-m deep borhole hirizontally drilled at Mizunami Underground Labotatory. Previously, sulfate redution rates of $1-5 \mathrm{nM} \mathrm{yr}^{-1}$ have been biogeochemically estimaed, which agree with wellcharacterized deep biospheres [2]. Unexpectedly, the amendement of $0.1 \mathrm{M}$ sulfate without the addition of any energy sources resulted in doubling of originally dominant microbioal populations of Nitrospirae and Ignavibateriae within 7 days. It is also unexpected that the production of hydrogen sulfide was not observed during the microbial growth.

The Horonobe Underground Laboratory was constructed in tertiary sedimentary rocks, from which methae is economically produced for local communities. Laboratory high-pressure incubations were conducted for 215-m deep groundwater where anaerobic methane-oxidizing archaea subtype 2d (ANME-2d) with multi-heme cytochromes contaning 50 or more heme-biding motifs were dominant [3]. ${ }^{13} \mathrm{C}$-labelled methane was oxidized without adding electron acceptors such as amorphous Fe(III), nitrate and sulfate. Although suspened particles containing Fe(III) are suspected to serve as an electron acceptor, the production of aqueous $\mathrm{Fe}$ (II) was not evident. Low-pressure incubations led to neglegible oxidation of ${ }^{13} \mathrm{C}$-labelled methane coupled to substntial production of aqueous $\mathrm{Fe}(\mathrm{II})$. These results highlight pressure-dependent metabolic activities of subsurface microbiome.

This work has been supported in part by a grant from the Ministry of Economy, Trade and Industry (METI).

[1] Hehler \& Jørgensen (2013) Nature Rev. Microbiol 11, 83-94. [2] Suzuki et al. (2014) PLOS One, id. 0113063. [3] Hernsdorf et al. (2017) ISME J, 11,1915-1929. 